\title{
Dynamic System Sustainability Simulation Modelling
}

\author{
Normunds Mihailovs, Sarma Cakula \\ Vidzeme University of Applied Sciences
}

\section{INTRODUCTION}

Assessing the sustainability of dynamic, open and complex systems with many stakeholders, interrelated components and interactions and forecasting with traditional study methods is complicated and has its limitations. Therefore, often researchers, when forecasting the sustainability of a dynamic system, rely on subjective judgment without references to assessment standards.

The aim of the paper is to create an imitation model for the sustainability of a dynamic system in order to assess and forecast the sustainability of the system under alternative development scenarios. It includes 3 main aspects - how sustainable is the dynamic system, what is the level of sustainability of a dynamic system under alternative development scenarios and what additions are needed to improve the functioning of the imitation model.

The research question of the paper is: what imitation model can effectively analyse and forecast a dynamic system in the case of the tourism object Cesis Palace.

Sustainable development researchers offer to build on traditional principles and interlinked dimensions of sustainable development: environment, economic and social, and adapt them to the dynamic system, which is characterized by the interactions between components (Tanguay et.al., 2011; Mai, Smith, 2018). One type of study that helps explain such systems is simulation modelling, which is often used when researching the interaction of dynamic systems (Johnson, 2011).

\section{MATERIALS AND METHODS}

The Cesis Palace complex as a tourism site was used in the paper for an example of a dynamic system, since tourism is both a dynamic system with many interlinked components and equally important are the three dimensions of sustainability for its longterm development.

During the study, multiple data acquisition methods were used: a structured interview, analysis of statistics, case study analysis and an expert interview on the created imitation model.

To achieve the goal of the research, a model of a tourism sustainability imitation model was created using the STELLA dynamic system modelling environment. The model and the selection of indicators were based on the three key sustainability dimensions: economy, environment and society/culture.

\section{RESULTS}

The result of the work is a computer model that helps to assess the sustainability of a real-life system and its dimensions by entering data generated during the study. It concludes that the tourism object under consideration is potentially sustainable. Simulating alternative development scenarios, it can be concluded that the elements of one group of indicators can affect both the sustainability level of their own dimension, as well as the indicators of other dimensions and their sustainability level, as well the sustainability of the system overall. Significant changes in the system take place in a situation where a number of indicator groups are affected by the changes.

\section{DISCUSION AND CONCLUSIONS}

In order to use this model further, it would be necessary to develop an improved methodology for evaluating model indicators.

To ensure a more efficient model performance and data quality, data security and integrity should be taken into account. Creation of an imitation model requires the acquisition, processing and issuing of large amounts of data that are exposed to data security and integrity risks, which may have a negative impact, not only on the functioning of the model, but also on the system itself.

To significantly improve the definition, selection, value assignment, and to more accurately identify the importance of interfacing elements and to express future forecasts, the author proposes to evaluate the use of machine-learning in imitation modelling. Machine-learning algorithms are increasingly used by researchers in mechanical engineering applications.

\section{KEYWORDS}

Dynamic system, simulation modelling, sustainability

\section{REFERENCES}

Johnson, P.A. (2011) Visioning local futures: agent-based modelling as a tourism planning support system. McGill University, Montreal.

Mai, T., Smith, C. (2018). Scenario-based planning for tourism development using system dynamic modelling: A case study of Cat Ba Island, Vietnam. Tourism Management, 68, 336-354.

Tanguay, G., Rajaonson, J., Therrien, M. (2011) Sustainable Tourism Indicators: Selection Criteria for Policy Implementation and Scientific Recognition. Scientific series, Montreal. ISSN 1198-8177, pp 1-20 